\title{
Bloodstream infections in haematological cancer patients colonized by multidrug-resistant bacteria
}

\author{
C. Cattaneo ${ }^{1}$ (D) R. Di Blasi ${ }^{2}$ - C. Skert ${ }^{3} \cdot$ A. Candoni ${ }^{4} \cdot$ B. Martino ${ }^{5} \cdot$ N. Di Renzo ${ }^{6} \cdot$ M. Delia $^{7} \cdot$ S. Ballanti ${ }^{8} \cdot$ F. Marchesi $^{9}$.

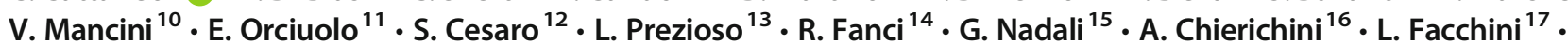 \\ M. Picardi ${ }^{18} \cdot$ M. Malagola $^{3} \cdot$ V. Orlando ${ }^{1} \cdot$ E. M. Trecarichi ${ }^{19} \cdot$ M. Tumbarello $^{19} \cdot$ F. Aversa $^{13} \cdot$ G. Rossi $^{1} \cdot$ L. Pagano $^{2} \cdot$ on \\ behalf of SEIFEM Group
}

Received: 10 January 2018 / Accepted: 21 April 2018 / Published online: 28 April 2018

(C) Springer-Verlag GmbH Germany, part of Springer Nature 2018

\begin{abstract}
Infections by multidrug-resistant (MDR) bacteria are a worrisome phenomenon in hematological patients. Data on the incidence of MDR colonization and related bloodstream infections (BSIs) in haematological patients are scarce. A multicentric prospective observational study was planned in 18 haematological institutions during a 6-month period. All patients showing MDR rectal colonization as well as occurrence of BSI at admission were recorded. One-hundred forty-four patients with MDR colonization were observed (6.5\% of 2226 admissions). Extended spectrum beta-lactamase (ESBL)producing (ESBL-P) enterobacteria were observed in 64/144 patients, carbapenem-resistant (CR) Gram-negative bacteria in 85/144 and vancomycin-resistant enterococci (VREs) in 9/144. Overall, 37 MDR-colonized patients (25.7\%) developed at least one BSI; 23 of them $(62.2 \%, 16 \%$ of the whole series) developed BSI by the same pathogen (MDRrel BSI), with a rate of $15.6 \%$ (10/64) for ESBL-P enterobacteria, $14.1 \%$ (12/85) for CR Gram-negative bacteria and 11.1\% (1/9) for VRE. In 20/23 cases, MDRrel BSI occurred during neutropenia. After a median follow-up of 80 days, 18 patients died (12.5\%). The 3-month overall survival was significantly lower for patients colonized with CR Gram-negative bacteria (83.6\%) and VRE (77.8\%) in comparison with those colonized with ESBL-P enterobacteria (96.8\%). CR-rel BSI and the presence of a
\end{abstract}

C. Cattaneo

chiara.cattaneo@libero.it

1 Haematology, Spedali Civili, 25123 Brescia, Italy

2 Institute of Haematology, Università Cattolica S. Cuore, Rome, Italy

3 Chair of Haematology, Unit of Blood Diseases and Stem Cell Transplantation, University of Brescia, Brescia, Italy

4 Division of Haematology and Stem Cell Transplantation, University Hospital of Udine, Udine, Italy

5 Haematology Unit, Bianchi-Melacrino-Morelli Hospital, Reggio Calabria, Italy

6 UOC di Ematologia e Trapianto di Cellule Staminali", PO "Vito Fazzi”, Lecce, Italy

7 Haematology Division, University of Bari, Bari, Italy

8 Institute of Haematology and Stem Cell transplantation, Ospedale Santa Maria della Misericordia, University of Perugia, Perugia, Italy

9 Haematology and Stem Cell Transplantation Unit, Regina Elena National Cancer Institute, Rome, Italy

10 Department of Haematology and Oncology, A.O. Ospedale Niguarda Cà Granda, Milan, Italy
11 Haematology Unit, Azienda Ospedaliero-Universitaria Pisana, Ospedale Santa Chiara, Pisa, Italy

12 Pediatric Haematology Oncology, Azienda Ospedaliera Universitaria Integrata, Verona, Italy

13 Haematology and BMT Unit, Azienda Ospedaliero-Universitaria di Parma, Parma, Italy

14 Haematology Unit, Careggi Hospital and University of Florence, Florence, Italy

15 Section of Hematology, Department of Clinical and Experimental Medicine, University of Verona, Verona, Italy

16 UOC Ematologia Azienda Ospedaliera S. Giovanni Addolorata, Rome, Italy

17 Haematology Unit, AO S. Maria Nuova, IRCCS, Reggio Emilia, Italy

18 Dipartimento di Scienze Biomediche Avanzate, Università Federico II Napoli, Naples, Italy

19 Institute of Infectious Disease, Università Cattolica S. Cuore, Rome, Italy 
urinary catheter were independent predictors of mortality. MDR rectal colonization occurs in $6.5 \%$ of haematological inpatients and predicts a $16 \%$ probability of MDRrel BSI, particularly during neutropenia, as well as a higher probability of unfavourable outcomes in CR-rel BSIs. Tailored empiric antibiotic treatment should be decided on the basis of colonization.

Keywords Haematologic patients $\cdot$ Multidrug-resistant bacteria $\cdot$ Colonization $\cdot$ Bloodstream infections

\section{Introduction}

Multidrug-resistant (MDR) bacteria, particularly Gramnegative (Gram-neg) strains, are a growing and worrisome phenomenon worldwide, with a demonstrated increase in mortality and public health costs [1]. As only few antibiotics at present are active against MDR bacteria, prevention is still considered the best strategy. This includes epidemiologic surveillance of colonization and infection and prompt isolation of cases. Identifying bacterial colonization may allow to better select empiric antibiotic treatment at the onset of fever or infection, and early adequate therapy obtains a better response in MDR infections [2, 3]. Gut decontamination is also a potential tool for preventing carbapenem-resistant (CR) enterobacteria infections, but its efficacy is often limited, and it may induce antibiotic resistance [4].

Haematological cancer patients are considered a population at risk for MDR infections because they experience prolonged antibiotic pressure and hospitalizations [5, 6]. Recently, in a retrospective Italian study, the incidence of CR Klebsiella pneumoniae infection was 0.4 and $2 \%$ in autologous and allogeneic stem cell transplantation (SCT), respectively. Infection-related mortality was $16 \%$ in autologous and $64.4 \%$ in allogeneic SCT [7]. Mortality caused by CR K. pneumoniae and MDR Pseudomonas aeruginosa was also unacceptably high among haematological cancer patients, ranging between 36 and 58\% [8,9]. Vancomycin-resistant enterococci (VREs) were associated with reduced survival in SCT recipients, although the attributable mortality was relatively low [10].

Available data concerning the real-life epidemiology of MDR bacterial strains among haematological patients are scarce, as are studies on the relationship between rectal colonization and infection, particularly the risk of bloodstream infections (BSIs), which has been analysed so far in few retrospective and prospective studies [11,12].

Therefore, a multicentric prospective observational study was conducted in the haematological setting within the SEIFEM (Sorveglianza Epidemiologica Infezioni Fungine in Emopatie Maligne) group to clarify the incidence of MDR bacterial colonization, the probability of developing MDR BSI in colonized patients and their outcomes.

\section{Patients and methods}

\section{Design of the study}

This prospective observational study was conducted by 18 haematological institutions participating in the SEIFEM. The study was approved by the ethics committee of each of the participating institutions, and informed consent was obtained from all patients for being included in the study. The procedures followed were in accordance with the ethical standards of the responsible committee on human experimentation (institutional and national) and with the Helsinki Declaration of 1975 , as revised in 2000.

During a 6-month period, between March 1 and August 31, 2015 , all patients with a haematological neoplasm admitted to 18 Italian centres participating in SEIFEM were screened at admission for MDR colonization with a rectal swab. Each hospital conducted screening rectal swab according to its own protocols. Rectal swabs were streaked onto selective agar; species identification was made, and antimicrobial susceptibility was tested by disk diffusion method. Both Gramneg and Gram-positive (Gram-pos) MDR colonizing bacteria were considered in the final analysis. Patients showing rectal MDR bacterial colonization were recorded in a database where the occurrence of any BSI was correlated with age, sex, type and phase of disease, stem cell transplantation, previous antibiotic treatment, presence of invasive devices, and type of colonizing bacteria. The follow-up was conducted for at least 1 month (range 30-270 days) after the date of known colonization.

\section{Definitions}

BSI was defined by the isolation of a bacterium in one blood culture; two positive cultures were required for diagnosing coagulase-negative staphylococci or Corynebacterium spp. BSI.

Antimicrobial resistance was defined according to the EUCAST clinical breakpoint [13]. Vancomycin resistance was considered with an MIC of $>4 \mathrm{mg} / \mathrm{L}$ for Enterococcus spp. and carbapenem resistance with an MIC of $>8$ for imipenem for Pseudomonas spp., Acinetobacter spp. and Enterobacteriaceae. Extended spectrum beta-lactamase 
Table 1 Characteristics of MDR colonized 144 patients

\begin{tabular}{|c|c|}
\hline Characteristics & Total $(n=144)$ \\
\hline Male/female ratio & $92 / 52$ \\
\hline Median age, years (range) & $60(0-89)$ \\
\hline Paediatric age $(<18, \%)$ & $7(4.9 \%)$ \\
\hline Age $\geq 60$ years $(\%)$ & $75(52 \%)$ \\
\hline \multicolumn{2}{|l|}{ Underlying haematological disease } \\
\hline Acute leukaemia $(\%)$ & $60(41.7 \%)$ \\
\hline Myeloid & 46 \\
\hline Lymphoblastic & 14 \\
\hline Lymphoma (\%) & $60(41.7 \%)$ \\
\hline Non-Hodgkin lymphoma & 51 \\
\hline Hodgkin lymphoma & 6 \\
\hline Chronic lymphocytic leukaemia & 3 \\
\hline Myeloma $(\%)$ & $17(11.8 \%)$ \\
\hline Myelodysplastic/myeloproliferative disease (\%) & $5(3.4 \%)$ \\
\hline Aplastic anaemia (\%) & $2(1.4 \%)$ \\
\hline \multicolumn{2}{|l|}{ Phase of haematological disease } \\
\hline Disease onset/induction chemotherapy (\%) & $37(25.7 \%)$ \\
\hline Complete or partial remission/consolidation chemotherapy (\%) & $68(47.2 \%)$ \\
\hline Relapsed or refractory disease/reinduction chemotherapy (\%) & $39(27.1 \%)$ \\
\hline \multicolumn{2}{|l|}{ Previous antibiotic treatment } \\
\hline Fluoroquinolone prophylaxis (\%) & $74(48.6 \%)$ \\
\hline Cephalosporin treatment $(\%)$ & $59(41 \%)$ \\
\hline Carbapenem treatment $(\%)$ & $49(34 \%)$ \\
\hline \multicolumn{2}{|l|}{ Presence of invasive devices } \\
\hline Central venous catheter $(\%)$ & $83(57.6 \%)$ \\
\hline Urinary catheter $(\%)$ & $25(13.9 \%)$ \\
\hline Autologous stem cell transplantation (\%) & $25(17.4 \%)$ \\
\hline Lymphoma $(\%)$ & $15(60 \%)$ \\
\hline Myeloma (\%) & $10(40 \%)$ \\
\hline Allogeneic stem cell transplantation (\%) & $15(10.4 \%)$ \\
\hline Acute myeloblastic leukaemia (\%) & $8(53.3 \%)$ \\
\hline Acute lymphoblastic leukaemia (\%) & $2(13.3 \%)$ \\
\hline Myeloma (\%) & $2(13.3 \%)$ \\
\hline Myelodysplastic/myeloproliferative disease (\%) & $2(13.3 \%)$ \\
\hline Severe aplastic anaemia $(\%)$ & $1(6.7 \%)$ \\
\hline
\end{tabular}

The chatacters in italic refers to subtype of disease
(ESBL) screening in Enterobacteriaceae was determined by the broth microdilution method (ESBL production if $\mathrm{a} \geq 8$ fold reduction is observed in the MIC of a cephalosporin, such as cefotaxime, ceftazidime or cefepime, combined with clavulanic acid than the MIC of cephalosporin alone) or by the combination disk method (cephalosporin alone and in combination with clavulanic acid; ESBL production if the inhibition zone diameter is $\geq 5 \mathrm{~mm}$ larger with clavulanic acid than without).

Bacteria showing resistance to at least one agent in three or more antimicrobial categories were defined as MDR [14].

\section{Statistical analysis}

Standard descriptive statistics, such as medians, ranges and proportions, were used to summarize the data. The $\chi^{2}$ test was used to compare differences in the percentage in a univariate analysis. Variables found to be significant $(p<0.1)$ in the univariate analysis were tested in a multivariate analysis, which was performed using a stepwise logistic regression model. Values of $p<0.05$ were considered statistically significant. Patient characteristics, type and phase of disease, presence of invasive devices and previous antibiotic treatment were analysed in relation to each type of MR colonization. 
Table 2 Incidence of different type of MDR bacteria colonization according to diagnosis and phase of haematological disease

\begin{tabular}{|c|c|c|c|}
\hline & $\begin{array}{l}\text { VRE } \\
(\%)\end{array}$ & $\begin{array}{l}\text { ESBL-P Enterobacteriaceae } \\
(\%)\end{array}$ & $\begin{array}{l}\text { CarbaR Gram-negative bacteria } \\
(\%)\end{array}$ \\
\hline \multicolumn{4}{|l|}{$\operatorname{AML}(n)$} \\
\hline Induction chemotherapy (292) & $0(-)$ & $3(1)$ & $8(2.7)$ \\
\hline $\begin{array}{l}\text { Consolidation chemotherapy } \\
\text { (303) }\end{array}$ & $4(1.3)$ & $7(2.3)$ & $11(3.6)$ \\
\hline Salvage chemotherapy (172) & $0(-)$ & $3(1.7)$ & $10(5.8)$ \\
\hline \multicolumn{4}{|l|}{$\operatorname{ALL}(n)$} \\
\hline Induction chemotherapy (65) & $0(-)$ & $1(1.5)$ & $1(1.5)$ \\
\hline $\begin{array}{l}\text { Consolidation chemotherapy } \\
\text { (111) }\end{array}$ & $0(-)$ & $5(4.5)$ & $5(4.5)$ \\
\hline Salvage chemotherapy (49) & $0(-)$ & $0(-)$ & $2(4.1)$ \\
\hline \multicolumn{4}{|l|}{ Lymphoma $(n)$} \\
\hline Disease onset (365) & $2(0.5)$ & $7(1.9)$ & $9(2.5)$ \\
\hline $\begin{array}{l}\text { Complete/partial remission } \\
\text { (339) }\end{array}$ & $2(0.6)$ & $14(4.1)$ & $12(3.5)$ \\
\hline $\begin{array}{l}\text { Relapse/refractory disease } \\
\text { (218) }\end{array}$ & $1(0.5)$ & $10(4.6)$ & $11(5)$ \\
\hline \multicolumn{4}{|l|}{ Myeloma $(n)$} \\
\hline Disease onset (20) & $0(-)$ & $1(5)$ & $1(5)$ \\
\hline $\begin{array}{l}\text { Complete/partial remission } \\
\text { (176) }\end{array}$ & $0(-)$ & $9(5.1)$ & $4(2.3)$ \\
\hline Relapse/refractory disease (60) & $0(-)$ & $1(1.7)$ & $3(5)$ \\
\hline
\end{tabular}

VRE vancomycin-resistant enterococci, ESBL-P extended spectrum beta lactamases producers, CarbaR carbapenem resistant, $A M L$ acute myeloid leukaemia, $A L L$ acute lymphoblastic leukaemia.
The above-mentioned variables, together with types of MDR colonization, stem cell transplantation and intensive care unit admission, were included in the analysis of all MR-related BSIs, ESBL-P-related BSIs, CR-related BSIs and VRErelated BSIs. In the 30-day mortality analysis, all types of MR-related BSIs were also considered. Overall survival (OS) rates were estimated according to the method of Kaplan and Meier, and comparison between the groups was done with log-rank test.

\section{Results}

\section{Epidemiology of colonization}

During the 6-month study period, 144 patients with MDR rectal colonization were observed. VREs were responsible for colonization in 9/144 (6.3\%) of ESBL-producing (ESBL-P) Enterobacteriaceae in 64/144 (44.4\%) and CR Gram-neg bacteria in $85 / 144(59 \%)$ patients, respectively. Fourteen patients showed both ESBL-P and CR Enterobacteriaceae colonizations.

The incidence of MDR rectal colonization was $6.5 \%$ of all admissions (144/2226), with a predominance of CR and ESBL-P bacteria in comparison with VRE (respectively: 85/ $2226,3.8 \%$ and $64 / 2226,2.9 \%$ vs $9 / 2226,0.4 \%$ of the entire cohort). The characteristics of MDR-colonized patients are summarized in Table 1.

We also evaluated the incidence according to diagnosis and phase of haematological disease (Table 2). Overall, the incidence of MDR colonization appeared to be lower at disease onset or during induction therapy.

\section{Incidence of BSI in MDR-colonized patients}

Overall, 37/144 patients (25.7\%) colonized with MDR bacteria developed at least one BSI during the period of observation; $23 / 37$ (62.2\%, corresponding to $16 \%$ [23/144] of the whole series) developed BSIs by the same pathogen (MDRrel BSI) (1 VRE, 9 ESBL-P Escherichia coli, 1 ESBL-P K. pneumoniae, $8 \mathrm{CR} K$. pneumoniae, $1 \mathrm{CR}$ Enterobacter aerogenes, $1 \mathrm{CR}$ $P$. aeruginosa and $2 \mathrm{CR}$ Acinetobacter spp.). The median time from MDR colonization to MDRrel BSI was 12 days (range $0-74)$. In $87 \%$ of the cases, MDRrel BSI occurred during neutropenia $(20 / 23)$.

The rate of MDRrel BSI according to the type of antibiotic resistance was $11.1 \%$ (1/9) for VRE colonization, $15.6 \%(10 /$ 64) for ESBL-P Enterobacteriaceae and 14.1\% (12/85) for CR Gram-neg bacteria. Among CR-colonizing Enterobacteriaceae, only $K$. pneumoniae and Enterobacter spp. were associated with MDRrel BSI $(8 / 37,21.6 \%$ and $1 / 5,20 \%$, respectively) (Table 3). 
Table 3 MDRrel and unrelated BSI according to type of colonising MDR bacteria

\begin{tabular}{|c|c|c|}
\hline Type of colonising MDR bacteria $(N)$ & MDRrel BSI (\%) & Unrelated BSI (\%) (type) \\
\hline VRE (9) & $1(11.1 \%)$ & $\begin{array}{l}1(11.1 \%) \\
1 \text { P. aeruginosa }\end{array}$ \\
\hline ESBL-P E. coli $(50)$ & $9(18 \%)$ & $\begin{array}{l}2(4 \%) \\
1 \text { S. hominis } \\
1 \text { S. epidermidis }\end{array}$ \\
\hline ESBL-P K. pneumoniae (6) & $1(16.7 \%)$ & $0(-)$ \\
\hline ESBL-P Enterobacter spp. (5) & $4(80 \%)$ & $0(-)$ \\
\hline CarbaR C. freundii (3) & $0(-)$ & $0(-)$ \\
\hline CarbaR Enterobacter spp. (5) & $1(20 \%)$ & $0(-)$ \\
\hline CarbaR E. coli (20) & $0(-)$ & $\begin{array}{l}3(15 \%) \\
1 \text { E. faecalis } \\
1 \text { S. epidermidis } \\
1 \text { P. aeruginosa }\end{array}$ \\
\hline CarbaR K. pneumoniae (37) & $8(21.6 \%)$ & $\begin{array}{l}7(18.9 \%) \\
1 \text { S. haemolyticus } \\
1 \text { Vancomycin-resistant E. faecalis } \\
2 \text { P. aeruginosa } \\
1 \text { MDR P. aeruginosa } \\
1 \text { K. pneumoniae } \\
1 \text { R. mucilaginosa }+ \text { C. albicans }\end{array}$ \\
\hline CarbaR M. morganii (2) & $0(-)$ & $0(-)$ \\
\hline CarbaR P. mirabilis (3) & $0(-)$ & $\begin{array}{l}1(33 \%) \\
1 \text { S. aureus }\end{array}$ \\
\hline MDR Acinetobacter spp. (9) & $2(22.2 \%)$ & $\begin{array}{l}1(11.1 \%) \\
1 \text { S. haemolyticus }+ \text { C. albicans }\end{array}$ \\
\hline MDR $P$. aeruginosa $(10)$ & $1(10 \%)$ & $\begin{array}{l}2(20 \%) \\
1 \text { S. epidermidis } \\
1 \text { CR K. pneumoniae }\end{array}$ \\
\hline
\end{tabular}

$M D R$ multidrug resistant, CarbaR carbapenem resistant

${ }^{\text {a }}$ Not carbapenem resistant
Unrelated BSIs were observed in 17/144 colonized patients (11.8\% of the whole series, corresponding to $45.9 \%$ of all patients developing a BSI), including 3 de-novo MDR BSIs (1 CR P. aeruginosa and 1 CR $K$. pneumoniae and 1 VRE, which have occurred respectively 19,31 and 8 days after screening admission) (Table 3). In 2/85 (2.4\%) patients with CR-Gram-neg colonization (CR $K$. pneumoniae and CR $P$. aeruginosa), a concomitant candidaemia was observed.

\section{Predictive factors for colonization and MDRrel BSI}

Among MDR-colonizing bacteria, a correlation with previous antibiotic exposure was observed only for VRE colonization, associated with cephalosporin treatment $(p=0.02$, chi-square test). A multivariate model to analyse the risk of VRE colonization could not be established due to the small sample size. Multivariate analysis was performed to analyse the risk of ESBL-P enterobacteria and CR Gram-neg colonization. No association with previous antibiotic exposure was found for ESBL-P and CR colonization. ESBL-P colonization was less frequent in patients with urinary catheters (OR 0.2, CI 0.080.7 ) and with acute leukaemia (OR 0.4, CI 0.2-0.8). Younger (<60 years) patients (OR 2.1, CI $1-4.2, p=0.04$ ) not undergoing autologous SCT (OR 2.7, CI 1.1-6.8) and with urinary catheter showed more frequent colonization by CR Gram-neg bacteria (OR 3, CI 1-8.8, $p=0.002$ ).

Multivariate analysis of predictor variables for developing MDRrel BSI revealed that having lymphoma or a responding haematological disease was protective (OR 0.2 , CI $0.06-0.6, p=0.007$ and OR 0.3, CI $0.1-0.9, p=0.03$, respectively). Table 4 summarizes the statistical analyses for MDRrel BSI.

Statistical analyses of predictor variables for the MDRrel BSI subtype was performed only for ESBL-P and CR rel BSI. ESBL-P colonization was strictly related to ESBL-P-rel BSI (OR 26, CI 3-207, $p=0.002$ ); CR-rel BSI was correlated with 
Table 4 Univariate and multivariate analyses for MDRrel BSI

$\begin{array}{ll}\text { Univariate analysis } & \text { Multivariate analys } \\ \%, p & \text { OR }(95 \% \mathrm{CI}), p\end{array}$

Diagnosis

- Acute leukaemia (Y vs N)

52 vs $40,0.26$

- Lymphoma (Y vs N)

17 vs $45,0.01$

$0.2(0.06-0.6), 0.007$

- Myeloma (Y vs N)

22 vs $10,0.11$

Age

- $\geq 60$ (Y vs N)

57 vs $50,0.59$

Sex

- Male (Y vs N)

57 vs $67,0.34$

- Female

Disease onset/induction phase

- Y vs N

43 vs $33,0.34$

26 vs $26,0.96$

Complete/partial remission

- Y vs N

30 vs $50,0.08$

0.3 (0.1-0.9), 0.03

Relapsed/refractory disease

- Y vs N

43 vs $25,0.07$

Previous Fq prophylaxis

- Yvs N

61 vs $49,0.32$

Previous cephalosporin tx

- Y vs N

40 vs $41,0.95$

Previous carbapenem tx

- Y vs N

26 vs $44,0.11$

CVC

- Y vs N

61 vs $58,0.79$

Urinary catheter

- Y vs N

22 vs $17,0.54$

Previous intensive care admission

- Yvs N

9 vs $6,0.60$

Type of MDR colonization

- VRE (Y vs N)

4 vs $7,0.68$

43 vs $45,0.68$

57 vs $60,0.79$

- CR Gram-neg (Y vs N)

SCT

- Autologous (Y vs N)

26 vs $16,0.25$

9 vs $11,0.77$

- Allogeneic (Y vs N)

The chatacters in italic refers to subtype of disease

$F q$ fluoroquinolone, $T x$ therapy, $C V C$ central venous catheter

relapsed/refractory haematological disease (OR 14.2, CI 2.7$74.7, p=0.002)$ and with the presence of a urinary catheter (OR 14.5, CI 2.4-87.9, $p=0.004$ ), whereas a diagnosis of lymphoma was protective for CR-rel BSI (OR 0.3, CI $0.002-0.4, p=0.007)$.

\section{Outcome of MDR colonized patients}

After a median follow-up of 80 days (range 30-270), 18/144 patients died (12.5\%). Survival data collected at day 80 were available for all the 144 patients. The 3-month OS of the entire cohort was $88.36 \% \pm 2.99 \mathrm{SE}$, being significantly lower in patients colonized with VRE $(77.78 \% \pm 13.86 \mathrm{SE})$ and CR Gram-neg $(83.57 \% \pm 4.79 \mathrm{SE})$ in comparison with those colonized with ESBL-P enterobacteria $(96.8 \% \pm 2.23 \mathrm{SE}, p=$ 0.033 and 0.02 , respectively) (Fig. 1a, b).

The 30-day mortality from MDR colonization was 10/144 (6.9\%) and was attributable to MDRrel BSI in 6/10 patients (60\%), specifically to CR-rel BSIs in 5 patients (3 CR K. pneumoniae, $1 \mathrm{CR}$ P. aeruginosa and $1 \mathrm{CR}$ Acinetobacter spp.) and to VRE-rel BSI in 1 patient. One de-novo CR P. aeruginosa BSI was fatal within 30 days. 


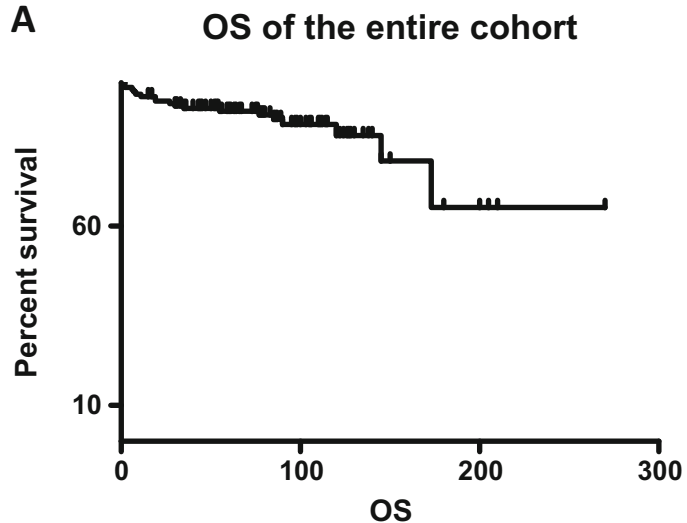

B

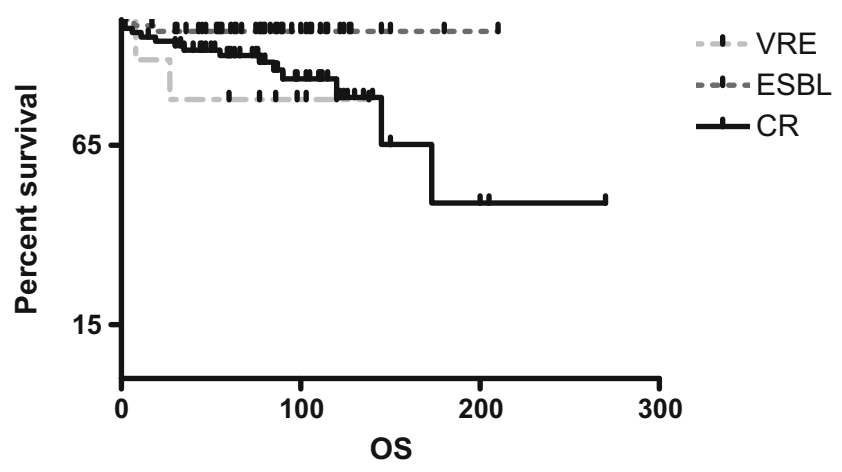

Fig. 1 Overall survival of 144 colonized patients (a) and according to type of colonization (b)

CR-rel BSI (OR 17.5, CI 3.5-86.4, $p=0.0004$ ) and the presence of a urinary catheter (OR 3.9, CI 1-17.2, $p=0.04$ ) were independent predictors of mortality at 30 days from MDR colonization, as revealed by the multivariate analysis (Table 5).

\section{Discussion}

In this large multicentre Italian survey, the incidence of MDR rectal colonization documented at admission and the probability of developing a related BSI was evaluated among all admitted patients with haematologic malignancies.

The overall incidence of MDR-colonized patients was $6.5 \%$, with a predominance of CR and ESBL-P bacteria in comparison with VRE, particularly during consolidation or salvage treatment. Incidence distribution showed a certain variability, ranging from 0 to $21 \%$ depending on the centre. The VRE and ESBL-P colonization frequency were lower in comparison with data reported by Liss et al. [15], which showed an incidence of 9.9 and $17.5 \%$, respectively. This discrepancy may be due to the different populations studied and to the different screening methods used. The German study included both oncological and haematological patients, and colonization was also evaluated in stool samples. Indeed, in a subsequent multicentre German study evaluating both rectal swabs and stool samples and performed in high risk haematologic patients, the incidence of ESBL-P enterobacteria colonization was $11.1 \%$ [12], but it was sharply lower (1.33-3.21\%) in a study considering only rectal swabs [16]. In a recent meta-analysis [17], showing an overall rate of colonization of $19 \%$, data reported from single studies analysed were highly variable depending from the different populations analysed and from their geographic origin, as well as from the screening methods used.

The rate of colonization by CR K. pneumoniae was $2.6 \%$ in our study, slightly higher than that reported by Girmenia et al. in SCT [7], confirming that this is a growing problem in endemic areas, such as Italy.

The predictive factors for MDR colonization are difficult to identify, and our study is not primarily designed to this end. In particular, the impact of previous antibiotic prophylaxis or other antibiotic treatment could not be evaluated, as data on non-colonized patients were not available. However, considering CR Gram-neg colonization, commonly regarded as a marker of illness severity, it was more frequent in patients of younger age, carrying a urinary catheter and undergoing treatments different from autologous SCT.

In our study, MDRrel BSIs were observed in $16 \%$ of colonized patients, representing approximately two thirds of all BSIs observed in this setting of patients. The probability of MDRrel BSI was similar for different types of MDR bacteria, confirming the propensity to develop a BSI, regardless of the type of colonizing bacteria. Unrelated BSIs occurred in $11.8 \%$ of MDR-colonized patients, with both Gram-pos and Gramneg bacterial BSIs. Notably, the only two cases of candidaemia occurred in CR Gram-neg bacteria colonized patients.

The rate of VRE- and ESBL-P-BSIs in colonized patients reported is variable. In our study, it was similar to those reported by Zaas and Cornejo-Juárez [18, 19] but much higher than in other studies $[12,15]$. Notably, only K. pneumoniae and E. aerogenes, among $\mathrm{CR}$ enterobacteria, were responsible for MDRrel BSIs. This finding may be explained by a higher virulence of Klebsiella and Enterobacter genera in comparison with other enterobacteria. The rate of MDRrel BSIs in CR K. pneumoniae-colonized patients (21.6\%) was comparable in our study with the approximately $30 \%$ incidence reported by Girmenia et al. [7], considering the type of population in our study, which includes only 15 allogeneic SCT patients. These data confirm the importance of a tailored empiric antibiotic treatment in MDR colonized febrile patients, particularly during neutropenia. On the other hand, non-colonized patients, under conditions of clinical stability, should not receive empirically ESBL- or CR-active agents.

Data concerning the predictive factors of MDRrel BSIs among haematologic patients are scant. Our study showed that both a diagnosis of lymphoma and a controlled haematological disease were protective. Therefore, these groups of MDR- 
Table 5 Univariate and multivariate analysis for 30-day mortality from known MDR colonization
Univariate analysis

$\%, p$

Multivariate analysis

OR (95\% CI), $p$

Diagnosis

- Acute leukaemia (Y vs N)

30 vs $43,0.44$

- Lymphoma (Y vs N)

- Myeloma (Y vs N)

50 vs $40,0.55$

10 vs $12,0.85$

Age

- $\geq 60($ Y vs N)

70 vs $50,0.22$

Sex

- Male (Y vs N)

- Female

60 vs $66,0.72$

40 vs $34,0.72$

Disease onset/induction phase

- Y vs N

50 vs $24,0.07$

Complete/partial remission

- Y vs N

10 vs 50, 0.01

Relapsed/refractory disease

- Y vs N

40 vs $27,0.37$

Previous Fq prophylaxis

- Y vs N

60 vs $51,0.57$

Previous cephalosporin tx

- Y vs N

40 vs $41,0.95$

Previous carbapenem tx

- Y vs N

50 vs $33,0.27$

CVC

- Y vs N

40 vs $60,0.22$

Urinary catheter

- Y vs N

50 vs $15,0.005$

$3.9(1-17.2)$

Type of MDR colonization

- VRE (Y vs N)

- ESBL-P enterobacteria (Y vs N)

20 vs $5,0.08$

10 vs $47,0.02$

80 vs $57,0.16$

SCT

- Autologous (Y vs N)

0 vs $15,0.13$

10 vs $10,0.96$

60 vs 13, <0.0001

10 vs $0,0.0002$

0 vs $8,0.37$

50 vs $5,<0.0001$

$17.5(3.5-86.4), 0.0004$

- Y vs N

10 vs $12,0.85$

- Y vs N

Unrelated MDR BSI

- Y vs N

0 vs $2,0.63$

The chatacters in italic refers to subtype of disease

$F q$ fluoroquinolone, $T x$ therapy, $C V C$ central venous catheter 
colonized patients may be considered at low risk of developing MDR-rel BSIs.

The 3-month OS was lower in patients colonized with CR Gram-neg bacteria or VREs than ESBL-P enterobacteria. None of the patients with ESBL-P-rel BSI died, and the 30-day mortality was lower in ESBL-P colonized patients (10 vs $47 \%, 0.02$ at the univariate analysis). This observation, together with an association of ESBL-P colonization with a diagnosis different from $\mathrm{AL}$ and with the absence of a urinary catheter, may prompt a re-evaluation of the clinical impact of ESBL-P Enterobacteriaceae in haematologic patients.

The MDRrel BSI-attributable 30-day mortality in CR and VRE was high (60\%), mainly due to CR-rel BSIs. The development of MDRrel BSI was the only predictive factor for death in colonized patients as revealed by multivariate analysis, together with the presence of a urinary catheter, confirming the severity of this condition, as was also reported in other series [20,21].

In conclusion, our multicentric study showed a relatively low incidence of MDR rectal colonization in haematological cancer patients. However, this condition predicts a probability of $16 \%$ of MDRrel BSI, particularly during neutropenic phases, and a higher percentage of unfavourable outcomes in case of CR-rel BSIs. Among CR enterobacteria, only Klebsiella and Enterobacter genera appear to have a propensity to develop related BSIs. CR Gram-neg colonization configures a poor prognostic setting in comparison with ESBL-P enterobacteria colonization, which appears to be a less severe condition in haematologic cancer patients. Taken together, these data confirm that the choice of empiric antibiotic treatment should be guided by known colonization in haematologic patients.

Acknowledgments On behalf of SEIFEM Group, Angela Passi, Doriana Gramegna (Haematology, Spedali Civili, Brescia, Italy); Domenico Russo (Chair of Haematology, Unit of Blood Diseases and Stem Cell Transplantation, University of Brescia, Brescia, Italy); Davide Lazzarotto (Division of Haematology and Stem Cell Transplantation, University Hospital of Udine, Udine, Italy); Domenico Rotilio (Haematology Unit, Bianchi-Melacrino-Morelli Hospital, Reggio Calabria, Italy); Maria Rosaria De Paolis, UOC di Ematologia e Trapianto di Cellule Staminali", PO "Vito Fazzi" Lecce); Edoardo Simonetti, Maria Alessandra Innocente (Institute of Haematology and Stem Cell transplantation, Ospedale Santa Maria della Misericordia, University of Perugia, Italy); Antonio Spadea (Haematology and Stem Cell Transplantation Unit, Regina Elena National Cancer Institute, Rome, Italy); Francesco Mazziotta (Haematology Unit, Azienda Ospedaliero-Universitaria Pisana, Ospedale Santa Chiara, Pisa, Italy); Anna Pegoraro (Pediatric Haematology Oncology), Giuliana Lo Cascio (Microbiology and Virology) (Azienda Ospedaliera Universitaria integrata, Verona, Italy); Angelica Spolzino (Haematology and BMT Unit, Azienda Ospedaliero-Universitaria di Parma, Parma, Italy); Gloria Turri (Section of Haematology, Department of Clinical and Experimental Medicine, University of Verona, Italy); Barbara Veggia (UOC Ematologia Azienda Ospedaliera S. Giovanni Addolorata, Rome, Italy).

\section{Compliance with ethical standards}

The study was approved by the ethics committee of each of the participating institutions, and informed consent was obtained from all patients for being included in the study. The procedures followed were in accordance with the ethical standards of the responsible committee on human experimentation (institutional and national) and with the Helsinki Declaration of 1975 , as revised in 2000.

Conflict of interest The authors declare that they have no conflict of interest.

\section{References}

1. Rice LB (2008) Federal funding for the study of antimicrobial resistance in nosocomial pathogens: no ESKAPE. J Infect Dis 197(8): 1079-1081. https://doi.org/10.1086/533452

2. Zarkotou O, Pournaras S, Tselioti P, Dragoumanos V, Pitiriga V, Ranellou K, Prekates A, Themeli-Digalaki K, Tsakris A (2011) Predictors of mortality in patients with bloodstream infections caused by KPC-producing Klebsiella pneumoniae and impact of appropriate antimicrobial treatment. Clin Microbiol Infect 17(12): 1798-1803. https://doi.org/10.1111/j.1469-0691.2011.03514

3. Tumbarello M, Viale P, Viscoli C, Trecarichi EM, Tumietto F, Marchese A, Spanu T, Ambretti S, Ginocchio F, Cristini F, Losito AR, Tedeschi S, Cauda R, Bassetti M (2012) Predictors of mortality in bloodstream infections caused by Klebsiella pneumoniae carbapenemase-producing K. pneumoniae: importance of combination therapy. Clin Infect Dis 55(7):943-950. https://doi.org/10. 1093/cid/cis588

4. Girmenia C, Viscoli C, Piciocchi A, Cudillo L, Botti S, Errico A, Sarmati L, Ciceri F, Locatelli F, Giannella M, Bassetti M, Tascini C, Lombardini L, Majolino I, Farina C, Luzzaro F, Rossolini GM, Rambaldi A (2015) Management of carbapenem resistant Klebsiella pneumoniae infections in stem cell transplant recipients: an Italian multidisciplinary consensus statement. Haematologica 100(9):e373-e376. https://doi.org/10.3324/haematol.2015

5. Gudiol C, Tubau F, Calatayud L, Garcia-Vidal C, Cisnal M, Sánchez-Ortega I, Duarte R, Calvo M, Carratalà J (2011) Bacteraemia due to multidrug-resistant Gram-negative bacilli in cancer patients: risk factors, antibiotic therapy and outcomes. J Antimicrob Chemother 66(3):657-663. https://doi.org/10.1093/ $\mathrm{jac} / \mathrm{dkq} 494$

6. Rodríguez-Baño J, Picón E, Hernández JR, Cisneros JM, Peña C, Almela M, Almirante B, Grill F, Colomina J, Molinos S, Oliver A, Fernández-Mazarrasa C, Navarro G, Coloma A, López-Cerero L, Pascual A (2010) Risk factors and prognosis of nosocomial bloodstream infections caused by extended-spectrum-beta-lactamaseproducing Escherichia coli. J Clin Microbiol 48(5):1726-1731. https://doi.org/10.1128/JCM.02353-09

7. Girmenia C, Rossolini GM, Piciocchi A, Bertaina A, Pisapia G, Pastore D, Sica S, Severino A, Cudillo L, Ciceri F, Scimè R, Lombardini L, Viscoli C, Rambaldi A (2015) Infections by carbapenem-resistant Klebsiella pneumoniae in SCT recipients: a nationwide retrospective survey from Italy. Bone Marrow Transplant 50(2):282-288. https://doi.org/10.1038/bmt.2014.231

8. Pagano L, Caira M, Trecarichi EM, Spanu T, Di Blasi R, Sica S, Sanguinetti M, Tumbarello M (2014) Carbapenemase-producing Klebsiella pneumoniae and hematologic malignancies. Emerg Infect Dis 20(7):1235-1236. https://doi.org/10.3201/eid2007. 130094

9. Trecarichi EM, Pagano L, Candoni A, Pastore D, Cattaneo C, Fanci R, Nosari A, Caira M, Spadea A, Busca A, Vianelli N, Tumbarello 
M (2015) Current epidemiology and antimicrobial resistance data for bacterial bloodstream infections in patients with hematologic malignancies: an Italian multicentre prospective survey. Clin Microbiol Infect 21(4):337-343. https://doi.org/10.1016/j.cmi. 2014.11.022

10. Weinstock DM, Conlon M, Iovino C, Aubrey T, Gudiol C, Riedel E, Young JW, Kiehn TE, Zuccotti G (2007) Colonization, bloodstream infection, and mortality caused by vancomycin-resistant enterococcus early after allogeneic hematopoietic stem cell transplant. Biol Blood Marrow Transplant 13(5):615-621

11. Gedik H, Yıldırmak T, Simșek F, Kantürk A, Arıca D, Aydın D, Yokuș O, Demirel N, Arabacı C (2014) Vancomycin-resistant enterococci colonization and bacteremia in patients with hematological malignancies. J Infect Dev Ctries 8(9):1113-1118. https://doi. org/10.4314/ahs.v14i4.18

12. Vehreschild MJ, Hamprecht A, Peterson L, Schubert S, Häntschel M, Peter S, Schafhausen P, Rohde H, Lilienfeld-Toal MV, Bekeredjian-Ding I, Libam J, Hellmich M, Vehreschild JJ, Cornely OA, Seifert H (2014) A multicenter cohort study on colonization and infection with ESBL-producing Enterobacteriaceae in high-risk patients with haematological malignancies. J Antimicrob Chemother 69(12):3387-3392. https://doi.org/10.1093/jac/dku305

13. EUCAST: http://www.eucast.org/clinical breakpoints/

14. Magiorakos AP, Srinivasan A, Carey RB, Carmeli Y, Falagas ME, Giske CG, Harbarth S, Hindler JF, Kahlmeter G, Olsson-Liljequist B, Paterson DL, Rice LB, Stelling J, Struelens MJ, Vatopoulos A, Weber JT, Monnet DL (2012) Multidrug-resistant, extensively drug-resistant and pandrug-resistant bacteria: an international expert proposal for interim standard definitions for acquired resistance. Clin Microbiol Infect 18:268-281. https://doi.org/10.1111/j. 1469-0691.2011.03570.x

15. Liss BJ, Vehreschild JJ, Cornely OA, Hallek M, Fätkenheuer G, Wisplinghoff H, Seifert H, Vehreschild MJ (2012) Intestinal colonisation and blood stream infections due to vancomycin-resistant enterococci (VRE) and extended-spectrum beta-lactamase-producing Enterobacteriaceae (ESBLE) in patients with haematological and oncological malignancies. Infection 40(6):613-619. https://doi.org/ 10.1007/s15010-012-0269-y

16. Reddy P, Malczynski M, Obias A, Reiner S, Jin N, Huang J, Noskin GA, Zembower T (2007) Screening for extended-spectrum betalactamase-producing Enterobacteriaceae among high-risk patients and rates of subsequent bacteremia. Clin Infect Dis 45(7):846-852

17. Alevizakos M, Karanika S, Detsis M, Mylonakis E (2016) Colonisation with extended-spectrum $\beta$-lactamase-producing Enterobacteriaceae and risk for infection among patients with solid or haematological malignancy: a systematic review and meta-analysis. Int J Antimicrob Agents 48(6):647-654. https://doi.org/10. 1016/j.jjantimicag.2016.08.021

18. Zaas AK, Song X, Tucker P, Perl TM (2002) Risk factors for development of vancomycin-resistant enterococcal bloodstream infection in patients with cancer who are colonized with vancomycin-resistant enterococci. Clin Infect Dis 35(10):11391146

19. Cornejo-Juárez P, Suárez-Cuenca JA, Volkow-Fernández P, SilvaSánchez J, Barrios-Camacho H, Nájera-León E, Velázquez-Acosta C, Vilar-Compte D (2016) Fecal ESBL Escherichia coli carriage as a risk factor for bacteremia in patients with hematological malignancies. Support Care Cancer 24(1):253-259

20. Cattaneo C, Zappasodi P, Mancini V, Annaloro C, Pavesi F, Skert C, Ferrario A, Todisco E, Saccà V, Verga L, Passi A, Da Vià M, Ferrari S, Mometto G, Petullà M, Nosari A, Rossi G (2016) Emerging resistant bacteria strains in bloodstream infections of acute leukaemia patients: results of a prospective study by the Rete Ematologica Lombarda (Rel). Ann Hematol 95(12):1955-1963

21. Trecarichi EM, Pagano L, Martino B, Candoni A, Di Blasi R, Nadali G, Fianchi L, Delia M, Sica S, Perriello V, Busca A, Aversa F, Fanci R, Melillo L, Lessi F, Del Principe MI, Cattaneo C, Tumbarello M (2016) Bloodstream infections caused by Klebsiella pneumoniae in onco-hematological patients: clinical impact of carbapenem resistance in a multicentre prospective survey. Am J Hematol 91(11):1076-1081. https://doi.org/10.1002/ajh. 24489 